Pacific Journal of Mathematics

ON A NEW RADICAL IN A TOPOLOGICAL RING 


\title{
ON A NEW RADICAL IN A TOPOLOGICAL RING
}

\author{
R. A. MASSAGLI
}

The radical which is referred to in this paper was treated extensively by Wright in the case of topological groups. The present course of attack here is threefold: (1) to show the proximity of large powers of topologically nilpotent elements to the radical in a topological ring, (2) to determine a nilpotence condition on the radical and (3) to characterize the radical of all locally compact simple rings without divisors of zero. For a topological group, the radical possesses little, if any, algebraic structure aside from being a subgroup of the group. Viewed as an additive subgroup of a topological ring $R$, it is shown that the radical is an ideal of $R$. Relative to the nilpotence of the radical, the additive group structure of locally compact connected Jacobson semi simple rings is established to within topological isomorphism.

In the final section the theorem on nilpotence is used to characterize the radical of locally compact simple rings having no zero divisors.

1. Preliminaries and definitions. Throughout this paper we adopt the notation and terminology of Wright [8]. All topological groups will be assumed to be Abelian and Hausdorff. For a topological group $G$, a maximal 0-proper open semigroup in $G$ is a subset $M \leqq G$ satisfying (1) $M$ is a semigroup in $G$, (2) $M$ is an open set in $G$, (3) $0 \notin M$, and (4) $M$ is maximal with respect to (1)-(3).

For subset $A \subseteq G$ we define $s(A)=\{x \in G \mid x+A \subseteq A\}$ and $b(A)=$ $s(A) \cap s(-A)$. If $M$ is a maximal 0 -proper open semigroup in $G$, $b(M)$ is a closed subgroup of $G$ and $G$ is the disjoint union of $\{M,-M, b(M)\}$. [8; Th. 3.3].

By the $W$-radical of $G$ we mean $\cap b(M)$ where the intersection is taken over all maximal 0-proper open semi group $M$ in $G$. We denote the $W$-radical by $T(G)$. If $G$ contains no 0-proper open semi groups, (for example if $G$ is finite, or more generally if $G$ is compact) $T(G)=G$ and $G$ is called a radical group. If $T(G)=(0)$ we say that $G$ is $W$-semi-simple.

In [8] it is shown that in a locally compact group $G, T(G)$ is a fully warrented hereditary radical [6]. Specifically it is shown that $T(T(G))=T(G), T(G / T(G))=(0)$ and if $H \subseteq G$ is a closed subgroup, $T(H)=H \cap T(G)$.

In general the $W$-radical of $G$ contains the radical $N$ of Iwasawa which is defined as the maximal compact connected subgroup of a locally compact group $G$ [4]. In the special case that $G$ is connected, both radicals coincide. 
2. Locally compact rings. The following result is of major consequence in this paper and establishes a justification for studying the $W$-radical in topological rings. Furthermore, in view of the remarks in the last section and the fact that $T(R)$ is an ideal of $R$, the term ring radical associated with $T(R)$ is fully justified [6].

THEOREM 2.1. In any topological ring $R, T(R)$ is an ideal of $R$. In particular, $T(R)$ is closed under left and right quasi-inverses.

Proof. We show that if $\phi: R \rightarrow R$ is any continuous group homomorphism, then $\phi(T(R)) \subseteq T(R)$.

Suppose $x \in \phi(T(R))$ so that $x=\phi(t)$ for some $t \in T(R)$. Assume there exists a maximal 0 -proper open semigroup $M$ in $R$ with $x \in M$. Then $\phi^{-1}(M)$ is a 0 -proper open semigroup in $R$ containing $t$. An application of Zorn's lemma yields a maximal 0-proper open semigroup $M^{*} \supseteqq \phi^{-1}(M)$. Thus $t \in M^{*}$ contradicting that $t \in T(R)$ since $R$ is a disjoint union of $\left\{M^{*},-M^{*}, b\left(M^{*}\right)\right\}$. It follows that $x \in T(R)$ and the assertion is established.

To see that $T(R)$ is an ideal of $R$, let $x \in R$ be fixed, but arbitrary, are consider the continuous group homomorphism $\phi: R \rightarrow$ $R: r \rightarrow r x$. It follows that $r x \in T(R)$; similarly $x r \in T(R)$ and therefore $T(R)$ is a (two-sided) ideal of $R$.

The notion of topological nilpotence has a natural generalization to its algebraic analogue. In a sequel to this paper we shall see how topological nilpotence of elements plays a vital role in the characterization of the prime radical of $T(R)$ in a certain subclass of locally compact commutative rings. Here we develop some elementary properties relating topological nilpotence to the $W$-radical of a locally compact ring.

Definition 2.2. [5; p. 162]. Let $R$ be a topological ring. An element $x \in R$ is called topologically nilpotent in case $\lim x^{n}=0$.

Our first result along these lines shows that in locally compact rings with compact component of 0 , powers of topologically nilpotent elements are eventually in the $W$-radical. We begin by establishing the following lemma:

LEMMA 2.3. Let $R$ be locally compact totally disconnected ring. If $x \in R$ is topologically nilpotent, there exists $N \in Z^{+}$such that $n \geqq N$ implies $x^{n} \in T(R)$.

Proof. Assume $\lim x^{n}=0$. Since $R$ is locally compact and totally disconnected, find a compact open neighborhood $U$ of 0 . Choose $N \in \boldsymbol{Z}^{+}$so that $n \geqq N$ implies $n^{n} \in U$. (For $y \in R$ denote by 
$\langle y\rangle$ the additive subgroup generated by $\left.y_{0}\right)$ Now $\left\langle x^{n}\right\rangle \subseteq U$ and hence $\mathrm{Cl}\left\langle x^{n}\right\rangle \subseteq U$. The compactness of $U$ implies that $\mathrm{Cl}\left\langle x^{n}\right\rangle$ is compact, whence $\mathrm{Cl}\left\langle x^{n}\right\rangle \subseteq T(R)$ [8; Th. 8.10]. We conclude that $x^{n} \in T(R)$.

Throughout the remainder of this paper the symbol $K$ will be used to denote the connected component of 0 .

THEOREM 2.4. Let $R$ be a locally compact ring with $K$ compact. If $x \in R$ is topologically nilpotent, $x^{n} \in T(R)$ for all $n$ sufficiently large.

Proof. Since $K$ is closed, $R / K$ is locally compact and totally disconnected. If $\lim x^{n}=0$ for $x \in R$ it is easy to see that $\lim (x+K)^{n}=$ $K$. Applying 2.3 we have that $(x+K)^{n}=x^{n}+K \in T(R / K)$ for all $n$ sufficiently large. Since $K$ is compact, $K \subseteq T(R)$. From [8; Th. 4.7] we have $T(R / K)=T(R) / K$ whence, $(x+K)^{n} \in T(R) / K$. This shows that $x^{n} \in T(R)$.

We remark that the condition $K \cong T(R)$, which is insured by compactness of $K$, is emphatic in 2.4. The real numbers as a topological ring shows that the compactness of $K$ cannot be dropped from our assumptions. (For example, $1 / 2$ is topologically nilpotent, but $(1 / 2)^{n} \neq 0$ for any $n \geqq 1$.) Locally compact $W$ semi-simple rings with compact $K$ are necessarily totally disconnected. In such rings topological nilpotence is reduced (in fact is equivalent) to algebraic nilpotence. This comment is generalized in $\S 3$.

For the next few results we digress slightly to the study of the radical structure of locally compact abelian groups.

Wright [8] shows that in every topological abelian group $G$ there is a unique (closed) maximal radical subgroup which we denote by $H$. The symbol $\oplus \sum_{\alpha \in \approx} G_{\alpha}$ will denote the weak direct product of groups $G_{\alpha}, \alpha \in \mathscr{A}$, where $\mathscr{A}$ is an arbitrary index set.

Lemma 2.5. Let $G=\bigoplus \sum_{\alpha \in \mathscr{A}} G_{\alpha}$ where each $G_{\alpha}$ is a topological abelian group, and let $H_{\alpha}$ be the maximal radical subgroup of $G_{\alpha}$. Then $\bigoplus \sum_{\alpha \in \infty} H_{\alpha}$ is the maximal radical subgroup of $G$.

Proof. Define $\mathscr{S}=\left\{S \subseteq G \mid S \subseteq \bigoplus \sum_{\alpha \epsilon \mathscr{A}} H_{\alpha}\right.$ and $S$ is a radical subgroup\}. Partially order $\mathscr{S}$ by inclusion. If $\left\{S_{\beta}\right\}$ is a chain in $\mathscr{S}$, by $\left[8 ;\right.$ p. 483] it can be shown that $\cup S_{\beta} \in S$, whence $\left\{S_{\beta}\right\}$ is bounded above. Let $M \in \mathscr{S}$ be a maximal element. We show that $M=\bigoplus \sum_{\alpha \in \mathscr{M}} H_{\alpha}$. To see this, let $H=\bigoplus \sum_{\alpha \in \mathscr{Q}} H_{\alpha}$ and assume $M \neq H$. Then there exists $\alpha \in A$ with $M \neq M+H_{\alpha}$. Now $M+H_{\alpha}$ is $W$-radical since both $M$ and $H_{\alpha}$ are so; thus we have $M \neq M+H_{\alpha} \subseteq$ $\bigoplus \sum_{\alpha \in \ldots} H_{\alpha}$ contradicting the maximality of $M$. Therefore $M=H$. 
In every locally compact group the $W$-radical is the maximal radical subgroup of $G$ [8; Th. 8.10]. As a useful consequence to 2.5 we recite the following result whose proof is immediate.

THEOREM 2.6. Let $G=\bigoplus \sum_{\alpha \in \mathscr{A}} G_{\alpha}$ be a locally compact abelian group. Then $T(G)=\bigoplus \sum_{\alpha \in \mathscr{A}} T\left(G_{\alpha}\right)$.

We are now in a position to prove the most general result involving topologically nilpotent elements in a topological ring $R$ and the $W$-radical of $R$. In particular this result shows that in $W$-semisimple rings, topologically nilpotent elements approach their limit via points in Euclidean $n$-space. We designate by \|\| the Euclidean norm in Euclidean $n$-space.

THEOREM 2.7. Let $R$ be a locally compact ring and assume $x \in R$ is topologically nilpotent. Then given $M \in Z^{+}$there is an $n \in Z^{+}$such that for $K \geqq n, x^{k}=e_{k}+g_{k}$ where $g_{k} \in T(R)$ and $\left\|e_{k}\right\|<1 / M$.

Proof. As topological groups, $R=E^{n} \oplus G$ where $G$ is a group having compact component of 0 [3; Th. 24.30]. Assume $\lim x^{k}=$ $\lim \left(e_{k}+g_{k}\right)=0$, where $e_{k} \in E^{n}$ and $g_{k} \in G$ for $k \geqq 1$. It follows that $\lim e_{e}=\lim g_{k}=0$. Abiding by the argument of 2.3 and 2.4 it is plain that $g_{k} \in T(G)$ and $\left\|e_{k}\right\|<1 / M$ for large $k$. By $2.6 T(R)=$ $T\left(E^{n}\right) \oplus T(G)$, and since $T\left(E^{n}\right)=(0), T(G)$ is topologically isomorphic to $T(R)$ in which case the theorem obtains.

3. Locally compact rings with connected $W$-radical. This section constitutes the central theme of this paper. Here we formulate a sufficient condition to insure the algebraic nilpotence of the $W$-radical. Then by example we show the condition is not necessary. In conjunction with [8; Th. 8.3] our main theorem establishes up to topological isomorphism the group structure of connected locally compact Jacobson semisimple rings. This consequence may be compared to the following two results, the first of which follows trivially from 3.2 and [8; Th. 8.10]; the second of which is proved in [5; Th. 16].

Every compact connected Jacobson semisimple ring is (0).

A compact Jacobson semi simple ring in homeomorphic to a direct sum of finite simple rings.

We begin by reciting a well known result whose proof follows directly from the definition of a topological ring and the notion of a compact set.

Lemma 3.1. Let $R$ be a topological ring, and let $C \leqq R$ be a 
compact subset. Then given an open neighborhood $U$ of 0 there exists an open neighborhood $V$ of 0 such that $V \cdot C \subseteq U$.

THEOREM 3.2. Let $R$ be a locally compact ring with $T(R)$ connected. Then $T(R)$ is a nilpotent ideal of index at most two. In particular $T(R) \subseteq J(R)$.

Proof. By [3] it will suffice to show that if $T(R)$ is viewed as a topological abelian group, and if $f$ is an arbitrary character on $T(R), f\left(T^{2}(R)\right)=0$.

Assume $f$ is a character on $T(R)$. For each $x \in T(R)$ define $T_{x}=\{t \in T(R) \mid f(t \cdot \mathrm{Cl}(x))=0\}$, where $\mathrm{Cl}(x)$ denotes the closure of the additive subgroup generated by $x$. Let $T^{\prime}=\{a \in T(R) \mid f(a \cdot T(R))=0\}$. We show $\bigcap_{x \in T(R)} T_{x}=T^{\prime}$. If $y \in T^{\prime}$ and $x \in T(R)$ is arbitrarily chosen, then $f(y \cdot \mathrm{Cl}(x))=0$; hence $y \in T_{x}$ and therefore $y \in \bigcap T_{x}$ where $x \in T(R)$. Conversely, if $y \in \bigcap T_{x}$ with $x \in T(R)$, let $t \in T(R)$ be arbitrary. Then $f(y \cdot \mathrm{Cl}(t))=0$ since, in particular, $y \in T_{t}$; whence $f(y t)=0$. Thus $y \in T^{\prime}$. This establishes the claim. Next we show that for each $x \in T(R), T_{x}$ is an open subgroup of $T(R)$. It is plain that $T_{x}$ is a subgroup; we concentrate on the openness of $T_{x}$. It suffices to exhibit an open neighborhood of $T(R)$ which is contained in $T_{x}$. Since $f(0)=Z(Z$ is viewed as a point in $R / Z)$ and since $f$ is continuous find an open neighborhood $U$ of 0 (open in $T(R)$ ) such that $f(U) \cong(-1 / 4,1 / 4)+Z$. Since $x \in T(R), \mathrm{Cl}(x)$ is compact by [8; Th. 8.10]. By 3.1 there is an open neighborhood $V$ in $T(R)$ such that $V \cdot \mathrm{Cl}(x) \leqq U$. Now let $y \in V$ be arbitrary. Now since $n z \in \mathrm{Cl}(x)$ whenever $z \in \mathrm{Cl}(x)$, it follows that if $n \in Z^{+}$then $n y z \in U$.

Now suppose that for some $y \in V$ and some $z \in \mathrm{Cl}(x), f(y z)=r+Z$ with $r \in(-1 / 4,1 / 4)$. Then $f(n y z)=n f(y z)=n r+Z$; hence $n r \epsilon$ $(-1 / 4,1 / 4)$ for all $n \in Z^{+}$. It follows that $r=0$ and so $f(y z)=Z$. This argument shows that $V \subseteq T_{x}$ and thus $T_{x}$ is open in $T(R)$; since $T_{x}$ is a subgroup of $T(R), T_{x}$ is also closed. By assumption $T(R)$ is connected and so $T_{x}=T(R)$ for each $x \in T(R)$. It now follows that $f\left(T^{2}(R)\right)=0$ since $T^{\prime}=T(R)=\bigcap T_{x}$ where $x \in T(R)$. By Pontryagin duality, $T^{2}(R)=(0)$. Finally since $J(R)$ contains all nilpotent ideals, our theorem is established.

The next example shows that in locally compact rings, the connectedness of the $W$-radical is mandatory.

EXAMPLE. Let $Z[x]$ be the ring of polynomials over the integers and let $R=Z[x] /(2 x)$. Topologize $R$ with the discrete topology. Since $x+x \equiv 0(\bmod 2 x), x$ has torsion. Hence $T(R) \neq(0)$. Moreover, $T^{2}(R) \neq(0)$ because $x^{2} \not \equiv 0(\bmod 2 x)$. (Recall that $T(R)$ contains the additive torsion subgroup of $R$.) 
Nonconnected locally compact rings $R$ having the property that $T(R) \neq(0)$ and $T^{2}(R)=(0)$ are plentiful and easy to construct.

The following two results whose contents are undeniably known follow as corollaries to 3.2. We present them here since they are derived intrinsically from the $W$-radical.

COROLlaRY. [Cf. 2.] Every nontrivial compact ring $R$ containing no divisors of zero is totally disconnected.

Proof. We need only remark that $R$ is either connected or totally disconnected [5; p. 169]. The remainder of the proof follows directly from 3.2.

COROLlaRy. If $R$ is any topological ring with $K$ compact, then $K^{2}=(0)$.

Proof. Since $K$ is compact, $T(K)=K$; by $3.2 T^{2}(K)=K^{2}=(0)$.

The next result reveals an interesting interplay between algebraic nilpotence and $K$.

THEOREM 3.3. Let $R$ be a locally compact ring with $T(R)$ connected. If $K$ is compact, then topological nilpotence is equivalent to algebraic nilpotence. [Cf. to the remark following 2.4.]

Proof. Let $x \in R$ be topologically nilpotent so that $\lim x^{n}=0$. By $2.4 x^{n} \in T(R)$ for all $n$ sufficiently large. By $3.2\left(x^{n}\right)^{2}=x^{2 n}=0$; hence, $x$ is algebraically nilpotent. The converse implication is trivial.

The pathological behavior relative to the $W$-radical and the Jacobson radical is in general somewhat surprising. We conclude this section by citing three examples illustrating this peculiar behavior.

ExAMPLE. A topological ring $R$ in which $T(R)=(0)$ and $J(R)=R$.

Let $R^{\prime}$ be any ring with torsion subgroup (0), and let $R=J\left(R^{\prime}\right)$. Give $R$ the discrete topology. Then $J(R)=R$ and $T(R)=(0)$ [8; Th. 4.1].

ExAmple. A topological ring $R$ in which $T(R)=R$ and $J(R)=(0)$.

Let $R$ be any finite field with the discrete topology. Since $R$ is compact, $T(R)=R$; since $R$ is a field $J(R)=(0)$.

EXAMPLE. A topological ring $R$ in which $T(R) \neq J(R) \neq R$.

Let $R$ be the ring of formal power series over the real numbers $\boldsymbol{R}$. Consider $R$ as a discrete topological ring. It is well known that $J(R)=(x)$, the set of elements having constant term equal to 
zero. Now define a family of continuous real valued group homomorphisms $f_{n}: R \rightarrow R$ by $f_{n}\left(\sum a_{i} x^{i}\right)=a_{n}$. If $p(x)=\sum b_{i} x^{i} \in T(R)$ and $n \geqq 0$ is arbitrary, it follows by [9] that $f_{n}(p(x))=b_{n}=0$. Let $\left\{f_{\alpha}\right\}$ be the collection of all real valued continuous group homomorphisms on $R$ with $K_{\alpha}=\operatorname{ker} f_{a}^{\prime}$ for each $\alpha$. Then $T(R)=\bigcap K_{\alpha} \subseteq \bigcap_{n=1}^{\infty} K_{n}$ where $K_{n}=\operatorname{ker} f_{n}$ for each $n \geqq 1$. Hence, $p(x)=0$ and therefore,

$$
T(R)=(0) \varsubsetneqq(x)=J(R) \varsubsetneqq R \text {. }
$$

4. Locally compact simple rings. Locally compact non-discrete fields are completely characterized in the literature [7]. Using such a characterization one is easily able to classify those fields which are $W$-radical and those which are $W$-radical free. In this section we generalize this method of classification to locally compact simple rings containing no divisors of zero; we do this intrinsically without appealing to the inherent structure of the ring. Our main instrument here is Theorem 3.2. We begin by eliminating the discrete case.

THEOREM 4.1. For a discrete simple ring $R$ without divisors of zero, $T(R)=(0)$ if and only if Char $R=0$.

Proof. Assume $T(R) \neq(0)$ and pick a nonzero $x \in T(R)$. Since $R$ is simple, $T(R)=R$. By [8; Th. 4.1] $R$ is an additive torsion group and therefore has nonzero characteristic. Conversely, if $T(R)=$ (0) and if $x \in R$ is nonzero, then $n x \neq 0$ for all $n \in Z^{+}$since $T(R)$ contains the torsion subgroup of $R$. This shows that Char $R=0$.

Every locally compact ring without divisors of zero is either connected or totally disconnected [5; p. 169]. In view of this result and Theorem 4.1, our next theorem completely characterizes the $W$-radical of the rings in question.

THEOREM 4.2. Let $R$ be a nondiscrete locally compact simple ring without divisors of zero. Then

(1) If $R$ is connected, $T(R)=(0)$.

(2) If $R$ is totally disconnected, $T(R)=R$.

Proof. (1) Since $R$ is connected, so is $T(R)$; by $3.2 T^{2}(R)=(0)$. Since $R$ has no zero divisors, $T(R)=(0)$ obtains.

(2) If $R$ is totally disconnected, then $R$ contains a compact open subgroup $U$. Now since $U$ is compact, $U \subseteq T(R)(T(R)$ contains all compact subgroups). Finally, $R$ is nondiscrete implies $U \neq(0)$ whence, $T(R)=R$.

The author wishes to thank the referee for his helpful comments in the final revision of this paper. 


\section{REFERENCES}

1. N. Bourbaki, Elements of Mathematics, General Topology (Part I), Addison-Wesley, 1966.

2. J. E. Cude, Compact integral domains, Pacific J. Math., 32 (1970), 615-619.

3. Hewitt and Ross, Abstract Harmonic Analysis I, Academic Press, Inc., Pub., N. Y., 1963.

4. K. Iwasawa, Some types of topological Groups, Ann. of Math., 50 (1949), 507-558.

5. I. Kaplansky, Locally compact rings, Amer. J. Math., 69 (1970), 153-183.

6. W. G. Leavitt, General Theory of Radicals, Classroom Notes University of Nebraska, 1964.

7. Y. Otobe, On locally compact fields, Japanese, J. Math., 19 (1945), 189-202.

8. F. B. Wright, Topological abelian groups, Amer. J. Math., 79 (1957), 477-496.

9. F. Wright, J. Diem, Real characters and the redical of an abelian group, Trans. Amer. Math. Soc., 129 (1968), 517-529.

Received June 3, 1971 and in revised form November 10, 1972. This constitutes part of the author's dissertation written at the University of Nebraska-Lincoln under Professor M. C. Thornton.

UNIVERSITY OF NEBRASKA 


\section{PACIFIC JOURNAL OF MATHEMATICS}

\section{EDITORS}

\author{
H. SAMELson \\ Stanford University \\ Stanford, California 94305 \\ C. R. HоввY \\ University of Washington \\ Seattle, Washington 98105
}

J. DugundJI

Department of Mathematics

University of Southern California

Los Angeles, California 90007

RICHARD ARENS

University of California

Los Angeles, California 90024

\section{ASSOCIATE EDITORS}
E. F. BeCKENBACH
B. H. NEUMANN
F. WOLF
K. YoshidA

\section{SUPPORTING INSTITUTIONS}

UNIVERSITY OF BRITISH COLUMBIA

CALIFORNIA INSTITUTE OF TECHNOLOGY

UNIVERSITY OF CALIFORNIA

MONTANA STATE UNIVERSITY

UNIVERSITY OF NEVADA

NEW MEXICO STATE UNIVERSITY

OREGON STATE UNIVERSITY

UNIVERSITY OF OREGON

OSAKA UNIVERSITY

\author{
UNIVERSITY OF SOUTHERN CALIFORNIA \\ STANFORD UNIVERSITY \\ UNIVERSITY OF TOKYO \\ UNIVERSITY OF UTAH \\ WASHINGTON STATE UNIVERSITY \\ UNIVERSITY OF WASHINGTON

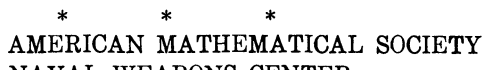 \\ NAVAL WEAPONS CENTER
}

The Supporting Institutions listed above contribute to the cost of publication of this Journal, but they are not owners or publishers and have no responsibility for its content or policies.

Mathematical papers intended for publication in the Pacific Journal of Mathematics should be in typed form or offset-reproduced, (not dittoed), double spaced with large margins. Underline Greek letters in red, German in green, and script in blue. The first paragraph or two must be capable of being used separately as a synopsis of the entire paper. The editorial "we" must not be used in the synopsis, and items of the bibliography should not be cited there unless absolutely necessary, in which case they must be identified by author and Journal, rather than by item number. Manuscripts, in duplicate if possible, may be sent to any one of the four editors. Please classify according to the scheme of Math. Rev. Index to Vol. 39. All other communications to the editors should be addressed to the managing editor, Richard Arens, University of California, Los Angeles, California, 90024.

50 reprints are provided free for each article; additional copies may be obtained at cost in multiples of 50 .

The Pacific Journal of Mathematics is issued monthly as of January 1966. Regular subscription rate: $\$ 48.00$ a year (6 Vols., 12 issues). Special rate: $\$ 24.00$ a year to individual members of supporting institutions.

Subscriptions, orders for back numbers, and changes of address should be sent to Pacific Journal of Mathematics, 103 Highland Boulevard, Berkeley, California, 94708.

PUBLISHED BY PACIFIC JOURNAL OF MATHEMATICS, A NON-PROFIT CORPORATION

Printed at Kokusai Bunken Insatsusha (International Academic Printing Co., Ltd.), 270, 3-chome Totsuka-cho, Shinjuku-ku, Tokyo 160, Japan. 


\section{Pacific Journal of Mathematics}

\section{Vol. 45, No. $2 \quad$ October, 1973}

Kenneth Paul Baclawski and Kenneth Kapp, Induced topologies for quasigroups and loops ............................................. 393

D. G. Bourgin, Fixed point and $\min -\max$ theorems $\ldots \ldots \ldots \ldots \ldots \ldots \ldots$

J. L. Brenner, Zolotarev's theorem on the Legendre symbol ............... 413

Jospeh Atkins Childress, Jr., Restricting isotopies of spheres .............. 415

John Edward Coury, Some results on lacunary Walsh series ................ 419

James B. Derr and N. P. Mukherjee, Generalized Sylow tower groups. II . . . . . . 427

Paul Frazier Duvall, Jr., Peter Fletcher and Robert Allen McCoy, Isotopy Galois

spaces .......................................... 435

Mary Rodriguez Embry, Strictly cyclic operator algebras on a Banach space ... 443

Abi (Abiadbollah) Fattahi, On generalizations of Sylow tower groups ......... 453

Burton I. Fein and Murray M. Schacher, Maximal subfields of tensor products . . 479

Ervin Fried and J. Sichler, Homomorphisms of commutative rings with unit

element .......................................... 485

Kenneth R. Goodearl, Essential products of nonsingular rings ............. 493

George Grätzer, Bjarni Jónsson and H. Lakser, The amalgamation property in

equational classes of modular lattices ...........................

507

$\mathrm{H}$. Groemer, On some mean values associated with a randomly selected simplex

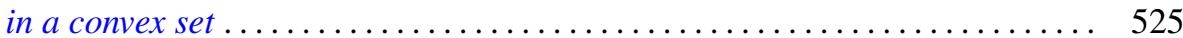

Marcel Herzog, Central 2-Sylow intersections .................... 535

Joel Saul Hillel, On the number of type-k translation-invariant groups ........ 539

Ronald Brian Kirk, A note on the Mackey topology for $\left(C^{b}(X)^{*}, C^{b}(X)\right) \ldots \ldots .543$

J. W. Lea, The peripherality of irreducible elements of lattice.............. 555

John Stewart Locker, Self-adjointness for multi-point differential operators ..... 561

Robert Patrick Martineau, Splitting of group representations ............... 571

Robert Massagli, On a new radical in a topological ring ................. 577

James Murdoch McPherson, Wild arcs in three-space. I. Families of Fox-Artin

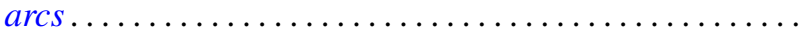

585

James Murdoch McPherson, Wild arcs in three-space. III. An invariant of

oriented local type for exceptional arcs . . . . . . . . . . . . ............ 599

Fred Richman, The constructive theory of countable abelian p-groups ........ 621

Edward Barry Saff and J. L. Walsh, On the convergence of rational functions

which interpolate in the roots of unity ..................

Harold Eugene Schlais, Non-aposyndesis and non-hereditary

decomposability..................................... 643

Mark Lawrence Teply, A class of divisible modules................... 653

Edward Joseph Tully, Jr., H-commutative semigroups in which each

homomorphism is uniquely determined by its kernel ................. 669

Garth William Warner, Jr., Zeta functions on the real general linear group ...... 681

Keith Yale, Cocyles with range $\{ \pm 1\} \ldots \ldots \ldots \ldots \ldots \ldots \ldots \ldots \ldots \ldots \ldots \ldots \ldots . \ldots \ldots$

Chi-Lin Yen, On the rest points of a nonlinear nonexpansive semigroup ........ 699 